\title{
Bus-Based VANET using ACO Multipath Routing Algorithm
}

Dr. R. Dhaya,

Department of Computer Science,

Sarat Abida Campus - King Khalid University,

KSA,

dhayave12005@gmail.com

Dr. R. Kanthavel,

Department of Computer Engineering,

King Khalid University,

KSA.

kanthavel2005@gmail.com

Abstract: Vehicular ad-hoc Network (VANET) for buses provides a good solution with inconsistent and high dynamic structure of a typical network. In this work, Street-centric routing algorithm is used for internet of energy for the bus-based VANET to optimize relay bus and route selection issues. As the initial step, we have used a multipath routing system that uses probability of path consistency and street consistency. This methodology is observed to positively improve end-to-end delay and also the packet delivery ratio. The next step is to optimize the characteristics of packet forwarding by means of an Ant Colony Optimization (ACO) methodology as the mechanism for relay-bus selection. The main goal of this paper is to transmit packets to the next forwarding relay with the help of relay-bus. On experimental analysis, the results indicate that there is a significant improvement in relay-bus choosing by reducing the end-to-end delay, cost of computation and unwanted beacon messages. Based on the analysis, we have arrived at the conclusion that the use of clustering and multipath will positively improve the performance of the proposed work.

Keywords: internet of energy; relay selection; multipath routing; clustering; Ant Colony Optimization 
Journal of trends in Computer Science and Smart technology (TCSST) (2021)

Vol.03/ No. 01

Pages: 40-48

https://www.irojournals.com/tcsst/

DOI: https://doi.org/10.36548/jtcsst.2021.1.004

\section{Introduction}

The aspect of disseminating information among vehicles by means of VANET is introduced in order to improve safety during travel on road as well as to prevent accidents from occurring. VANET is a database of intelligent vehicles that are used to make optimum usage of transportation data such as traffic light scheduling, traffic safety, traffic congestion detection etc, in order to decrease the occurrence of accidents on road. VANET is type of MANET which equips the vehicles with a means of communicating in a wireless fashion [1], establishing connectivity between them. This can be of many types such as vehicle-to-road, vehicle-to-internet, vehicle-to-vehicle and vehicle-to-sensor. To control and monitor the vehicular traffic in an intelligent manner, VANET serves an intelligent transport systems tool. This enhancement of the vehicles to smart vehicles paves way to high potential that leads to multiple services from road safety in traditional environment to smart communication in smart cities. Based on the term Internet of Things, the term Internet of Energy (IoE) has emerged which is widely used in industrial area to address the demands for effective utilization of energy production. The basic means of transportation that connects the city to every nook and corner route is buses. In order to cover the entire network of the city, the transportation department has laid down particular streets and lines. Accordingly, the buses will travel following a pre-defined departure interval and fixed arrival times [2]. The VANET is highly dynamic with respect to direction, location and speed. Because of the continuous service, uniform distribution of buses, fixed trajectories and broader coverage are some of the positive aspects of bus-based networks. Because of these advantages, a number of research works are being conducted on the bus-based VANET to improve the efficiency of data dissemination [3].

\section{Related Works}

Authors in [4] have proposed a bus-based VANET architecture which has two layers. The lower layer is composed of ordinary vehicles while the higher layer holds buses and road side units. Here the packets are forwarded with the help of buses that behave as relays, sending the packet to the destination. This work is commonly referred to as bus-based VANET because 
Journal of trends in Computer Science and Smart technology (TCSST) (2021)

Vol.03/ No. 01

Pages: 40-48

https://www.irojournals.com/tcsst/

DOI: https://doi.org/10.36548/jtcsst.2021.1.004

of the buses that are used as the major nodes in this methodology. The bus-based routing algorithm used in [5] establishes a path between the destination and the source using a multihop route. In particular, this methodology is built up of buses that are used to transmit the packets in a sequential manner. The packet is transmitted to the next hop on detecting the right relay that leads to the destination. However, if there are multiple bus lines involved, the packets will be forwarded at the overlapping point of the buses. Buses are used in this routing scheme methodology as a relay node and other vehicles will play the role of a receiver or a sender. In [6]. The researchers have introduced a novel technique, using buses as the routing backbone to build a bus-based routing technology that doesn't have an infrastructure. Using the real trajectory of buses, a protocol was formulated as a graph of bus lines. Moreover, the authors have also made use of the different communities into which the distributing bus lines are established. Using this lay out, it is observed that the busses that belong to a particular community will be able to communicate with each other in a quicker fashion.

Community based system further divides their routing methodology as intra and inter community routing. According to the frequency with which the buses communicate with each other, the relay buses are chosen such that delay during the routing process is decreased and subsequently, there is an improvement in the delivery ratio [7]. In [8], using the topology of the city streets, a bus-based street-centric routing strategy is followed. In this methodology, the traffic density, street between junctions and intersections were represented by weights, edges and vertices respectively. Two novel concepts namely probability of path consistency and probability of street consistency have been introduced. In this proposed work, the ACO algorithm is used to choose the relay-bus with the help of maximum link reliability metric [9]-[10]. The chosen relay-bus will be used to send the packets to the successive node without the need for using discovery process iteratively. Hence, there is a significant reduction in cost of the system in terms of time and energy. Moreover, we have incorporated a multipath routing stream that establishes a balance between the traffic data and will also provide the optimal relay path to follow, based on availability [11]. The proposed methodology is compared with GeoMob and CBS. The experimental analysis indicates that the use of ACO base clustering in SCRS has shown a positive improvement in the performance of the output. The paper is organised such that the next section shows the overview of the proposed 
Journal of trends in Computer Science and Smart technology (TCSST) (2021)

Vol.03/ No. 01

Pages: 40-48

https://www.irojournals.com/tcsst/

DOI: https://doi.org/10.36548/jtcsst.2021.1.004

algorithm and section 4 discusses the results obtained. Based on the experimental results, a conclusion is arrived upon in section 5.

\section{Proposed Work}

Consider a city road network with predefined bus lines and streets where there is uniform distribution of buses [12]. Fig.1 represents the architecture of the proposed bus-based VANET [13]. Here the messages are transmitted to and from the destination with the help of core nodes.

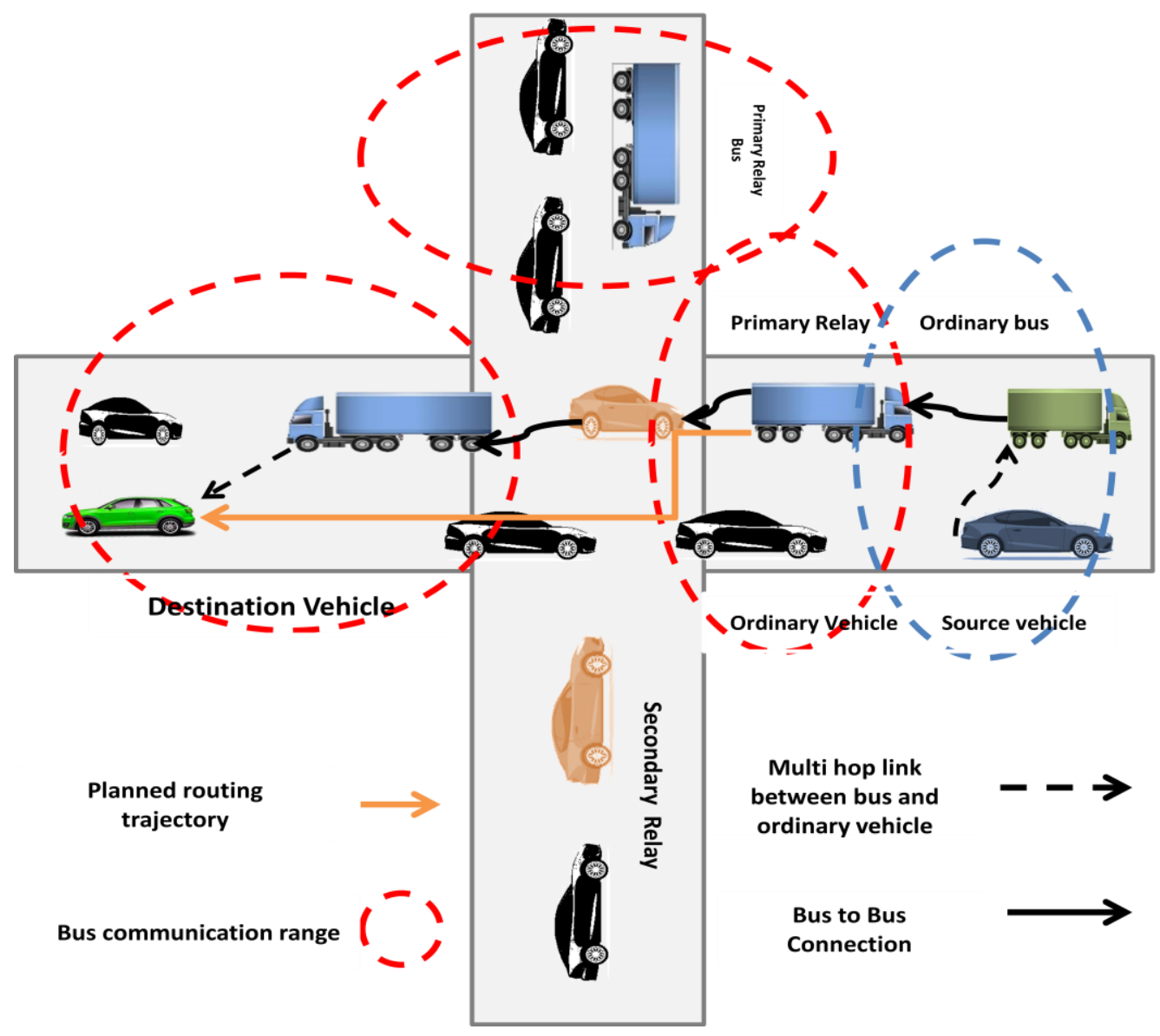

Fig.1. System Model of Proposed Methodology 
Journal of trends in Computer Science and Smart technology (TCSST) (2021)

Vol.03/ No. 01

Pages: 40-48

https://www.irojournals.com/tcsst/

DOI: https://doi.org/10.36548/jtcsst.2021.1.004

Every moving object is equipped with bus lines, digital maps, global positioning system and on-board units for the city. These buses serve as the basic means of routing to determine the next hop that will take the packet to the destination. The other moving objects can also be used as an alternative relay that can be used when the primary relay is not accessible or fails. Consider the radius of communication for the non-buses and buses to be $\mathrm{R}$. Then, based on the link availability, link reliability [14] can be determined with the help of Gaussian distribution such that:

$$
f(T)=\frac{R_{a b}}{\sigma \Delta v_{a b} \sqrt{2 \pi} T^{2}} e^{-\frac{\left(\frac{R_{a b}}{T}-x \Delta v_{a b}\right)^{2}}{\left(2 \sigma \Delta v_{a b}\right)^{2}}}
$$

Here $\sigma$ represents standard deviation and $x \Delta v_{a b}$ represents the mean. The probability of availability of the vehicles during the duration time $T_{\text {time }}$ can be expressed as:

$$
r_{t}(R)=\int_{t}^{t+T_{\text {time }}} f(T) d t \text { when } T_{\text {time }}>0
$$

where $r_{t}(R)$ represents the links' reliability at the time t.

There are three major parts of the routing algorithm:

- ACO-based clustering for forwarding relay-bus selection: The buses are used as the means to forward the packets, routing them to their destination. Each path that connects the two ends are made up of streets and the relay bus that corresponds to the selected street is chosen with the path $\mathrm{P}$ such that the complexity of the ACO algorithm can be expressed as $\mathrm{O}(\mathrm{n} * \mathrm{P}+\mathrm{Cof} * \mathrm{P})$.

- Street-centric routing algorithm for multiple route selection: Rather than the typical save and forward methodology, it involves carry and forward. In this paper, we make use of the multipath shortest-path selection. Accordingly, the probability of street consistency can be determined using the expression 
Journal of trends in Computer Science and Smart technology (TCSST) (2021)

Vol.03/ No. 01

Pages: 40-48

https://www.irojournals.com/tcsst/

DOI: https://doi.org/10.36548/jtcsst.2021.1.004

$$
P S C_{a, b}=\frac{n_{a, b}}{N_{b}} \cdot P S C_{b, a}=\frac{n_{a, b}}{N_{b}}
$$

- Routing Path using Bus-trajectories: Using the sub-map, it is possible to use the buses in order to cover the entire city. Using this routing algorithm, we can determine the probability with which the buses pass a particular bus-line that can be expressed as:

$$
\rho_{a, b}=\left\{\begin{array}{l}
\frac{1}{p_{a, b}}, \text { if } p_{a, b \neq 0} \\
\infty, \text { otherwise }
\end{array}\right.
$$

\section{Results and Discussion}

We have incorporated the proposed work using MATLAB for evaluating the results based on ACO clustering and link reliability. Fig. 1 represents the delivery ratio of the packets using the propose ACO based bus-VANET routing and other similar methodologies. It has been identified that the proposed methodology proves to provide a higher delivery ratio when compared with the others. Similarly, Fig.2. shows the average end-to-end delay where the proposed ACO with bus based-VANET proving to be better than other methodologies.

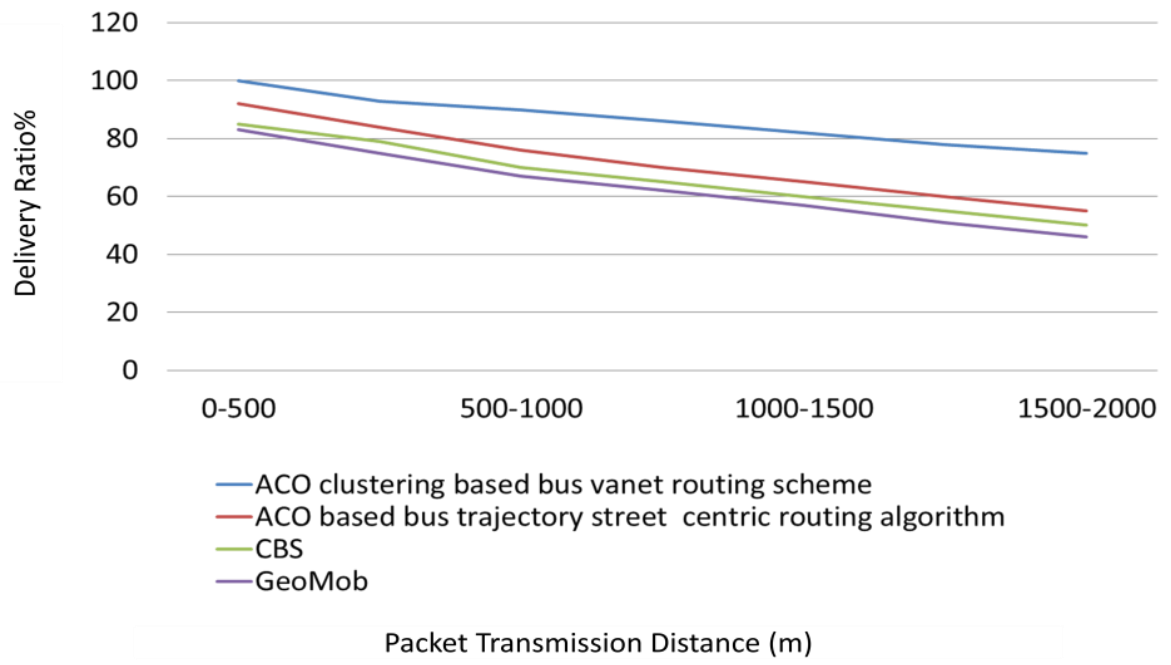

Fig.2. Packet Delivery Ratio based on Different Transmission Distances 
Journal of trends in Computer Science and Smart technology (TCSST) (2021)

Vol.03/ No. 01

Pages: 40-48

https://www.irojournals.com/tcsst/

DOI: https://doi.org/10.36548/jtcsst.2021.1.004

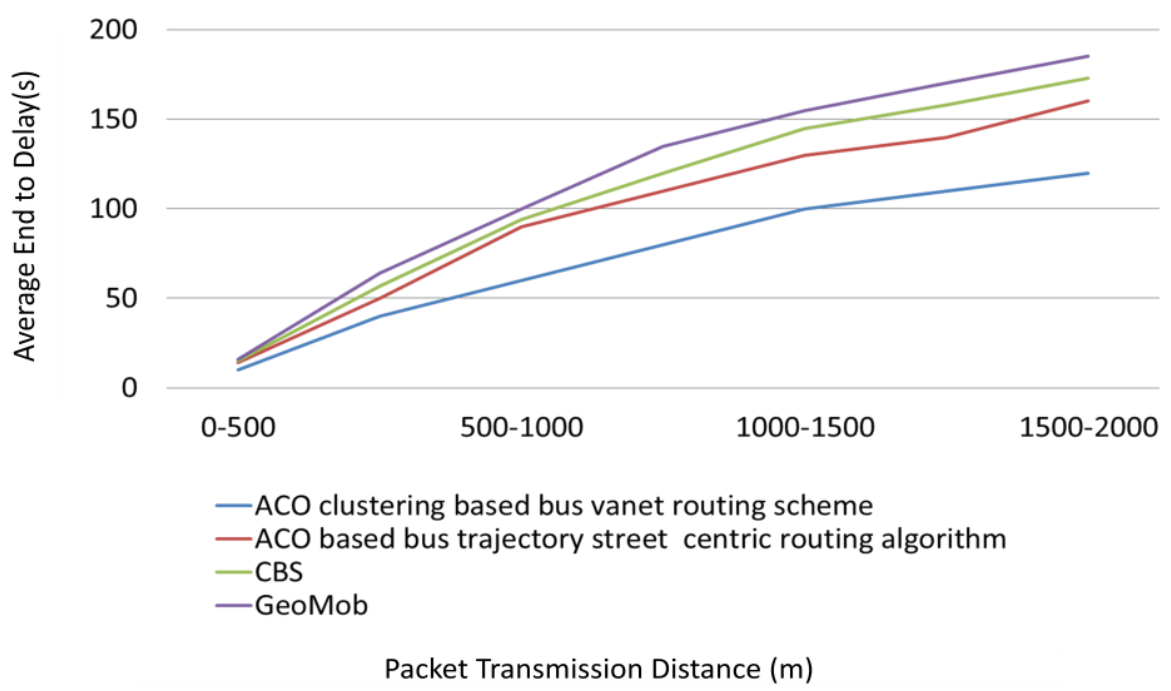

Fig.3. Average end-to-end delay based on Different Transmission Distances

Fig.4. indicates the comparison of experimental results observed for relay selection time with respect to different transmission distances.

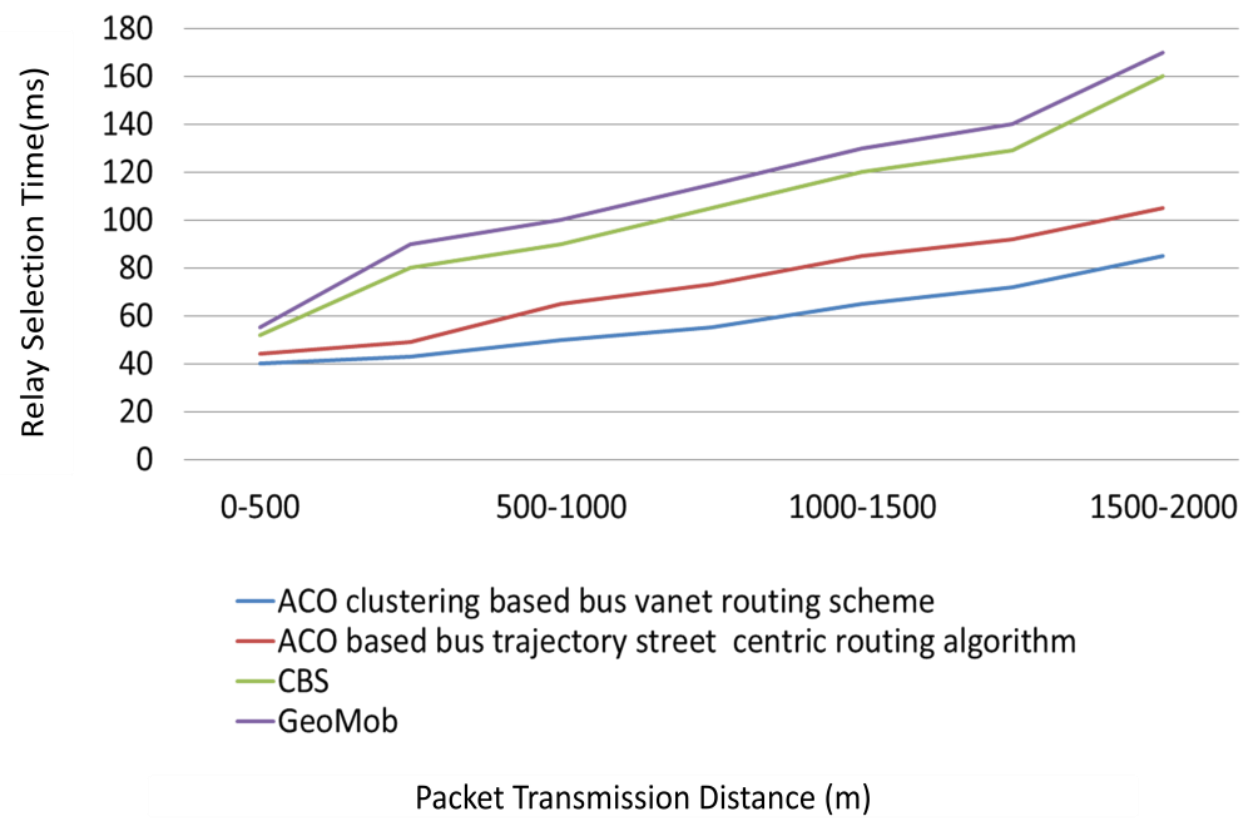

Fig.4. Relay bus-selection based on Different Transmission Distances 
Journal of trends in Computer Science and Smart technology (TCSST) (2021)

Vol.03/ No. 01

Pages: 40-48

https://www.irojournals.com/tcsst/

DOI: https://doi.org/10.36548/jtcsst.2021.1.004

\section{Conclusion}

The most crucial form of transportation that establishes a connection within the city by means of well-laid out roads are the buses. Using buses in VANET, we have proposed two major contributions in this paper. We have used multipath routing algorithm instead of uni-path routing algorithm used so far and it is observed to have improved the performance of the system, despite heavy traffic. We have also incorporated an ACO-based clustering algorithm for selecting the relay bus. Based on the results observed, we have arrived at the conclusion that this methodology operates in a manner similar to that of choosing cluster heads in clustering methods. It has been identified that there is a significant improvement in packet delivery ratio and end-to-end delay due to the use of ACO-based clustering. Hence the use of clustering as well as multipath method is said to be the optimal in our proposed methodology. It is also possible to use this methodology with similar mobility models. However, the output of the same will rely on the traceability of mobility. Though our proposed work observes an improved performance in terms of packet delivery ratio, E2E delay and time complexity, there is much work required upfront such as $\mathrm{CH}$ selection, cluster formation, inter-vehicles link reliability etc which will increase time complexity of setting up the system.

\section{References}

[1] Jiang, X., \& Du, D. H. (2015). Bus-vanet: A bus vehicular network integrated with traffic infrastructure. IEEE Intelligent Transportation Systems Magazine, 7(2), 47-57.

[2] Kitani, T., Shinkawa, T., Shibata, N., Yasumoto, K., Ito, M., \& Higashino, T. (2008, May). Efficient vanet-based traffic information sharing using buses on regular routes. In VTC Spring 2008-IEEE Vehicular Technology Conference (pp. 3031-3036). IEEE.

[3] Shi, J., Wang, X., Huang, M., Li, K., \& Das, S. K. (2017). Social-based routing scheme for fixed-line VANET. Computer Networks, 113, 230-243.

[4] Zhang, F., Liu, H., Leung, Y. W., Chu, X., \& Jin, B. (2016). CBS: Community-based bus system as routing backbone for vehicular ad hoc networks. IEEE Transactions on Mobile Computing, 16(8), 2132-2146. 
Journal of trends in Computer Science and Smart technology (TCSST) (2021)

Vol.03/ No. 01

Pages: 40-48

https://www.irojournals.com/tcsst/

DOI: https://doi.org/10.36548/jtcsst.2021.1.004

[5] Sivaganesan, D. (2019). Efficient routing protocol with collision avoidance in vehicular networks. Journal of Ubiquitous Computing and Communication Technologies (UCCT), 1(02), 76-86.

[6] Zhu, H., Fu, L., Xue, G., Zhu, Y., Li, M., \& Ni, L. M. (2010, March). Recognizing exponential inter-contact time in VANETs. In 2010 Proceedings IEEE INFOCOM (pp. 1-5). IEEE.

[7] Chaib, N., Oubbati, O. S., Bensaad, M. L., Lakas, A., Lorenz, P., \& Jamalipour, A. (2019). BRT: Bus-based routing technique in urban vehicular networks. IEEE Transactions on Intelligent Transportation Systems, 21(11), 4550-4562.

[8] Zhang, L., Jia, S., Liu, Z., Wang, Y., \& Liu, Y. (2015). Bus-Ads: Bus trajectory-based advertisement distribution in VANETs using coalition formation games. IEEE Systems Journal, 11(3), 1259-1268.

[9] Shirley, D. R. A. (2014, July). Systematic diagnosis of power switches. In 2014 International Conference on Embedded Systems (ICES) (pp. 32-34). IEEE.

[10] Srivastava, A., Prakash, A., \& Tripathi, R. (2020). Location based routing protocols in VANET: Issues and existing solutions. Vehicular Communications, 23, 100231.

[11] Satyajeet, D., Deshmukh, A. R., \& Dorle, S. S. (2016). Heterogeneous approaches for cluster based routing protocol in vehicular ad hoc network (vanet). International Journal of Computer Applications, 134(12), 1-8.

[12] Bhalaji, N. (2019). Performance evaluation of flying wireless network with Vanet routing protocol. Journal of ISMAC, 1(01), 56-71.

[13] He, J., Cai, L., Cheng, P., \& Pan, J. (2015). Delay minimization for data dissemination in large-scale VANETs with buses and taxis. IEEE Transactions on Mobile Computing, 15(8), 1939-1950.

[14] Sathesh, A. (2019). Optimized multi-objective routing for wireless communication with load balancing. Journal of trends in Computer Science and Smart technology (TCSST), 1(02), 106-120. 